\title{
BAS2 Is Required for Conidiation and Pathogenicity of Colletotrichum gloeosporioides from Hevea brasiliensis
}

\author{
Bang An * (1D), Wenfeng Wang, Yunfeng Guo, Qiannan Wang, Hongli Luo and Chaozu He * \\ Hainan Key Laboratory for Sustainable Utilization of Tropical Bioresources, Institute of Tropical Agriculture and \\ Forestry, Hainan University, Haikou 570228, China; wangwenfenghn@163.com (W.W.); \\ GUOYUNFENG18689731093@outlook.com (Y.G.); wangqiannan@hainu.edu.cn (Q.W.); \\ hlluo@hainu.edu.cn (H.L.) \\ * Correspondence: anbang@hainu.edu.cn (B.A.); czhe@hainu.edu.cn (C.H.); \\ Tel.: +86-898-6616-0728 (B.A. \& C.H.)
}

Received: 15 May 2018; Accepted: 13 June 2018; Published: 25 June 2018

\begin{abstract}
The hemibiotrophic fungi Colletotrichum gloeosporioides can cause anthracnose in rubber trees. By searching the genome of the fungal pathogen, the BAS2 encoding a biotrophy-associated secreted protein was identified. In the present study, the knockout mutants of $B A S 2$ were constructed and the functions of BAS2 were investigated. The in vitro assays showed that BAS2 was not necessary for vegetative growth but was important for normal asexual reproduction in C. gloeosporioides. Pathogenicity assays suggested that BAS2 was involved in the process of the pathogen penetrating into the host tissue. Subcellular localization analysis revealed that BAS2 showed secretional characteristics in the fungi, and BAS2 mainly function as a cytoplasmic protein after being secreted into the host cell. Extracellular proteomics analysis revealed that BAS2 was required for the secretion of a series of proteins, which were important for the pathogenicity of C. gloeosporioides. These data lead to a better understanding of the biotrophy-associated secreted protein in regulating the pathogenesis of C. gloeosporioides.
\end{abstract}

Keywords: Colletotrichum gloeosporioides; biotrophy-associated secreted protein; conidiation; pathogenicity; extracellular proteomics

\section{Introduction}

Anthracnose caused by the fungal pathogen Colletotrichum gloeosporioides is one of the main plant diseases in the rubber tree (Hevea brasiliensis), which leads to decreased rubber yield and serious economic losses to rubber planting. A hemibiotrophic lifestyle is a distinctive aspect of Colletotrichum species. After penetrating into the host epidermis, most Colletotrichum species initially colonize living plant tissue and obtain nutrients from living host cells. After a period of time, Colletotrichum switch to necrotrophy, leading to massive cell death and tissue destruction in the host. To overcome the plant's defense systems, plant pathogens employ effector proteins as molecular weapons to influence the physical processes of the host [1-3]. Genome and transcriptome analyses revealed that a series of effector proteins were induced before penetration and during biotrophy in Colletotrichum species [4]. It has been reported that the $C g D N 3$ gene [5] was implicated in the biotrophic phase and required for suppressing host resistance responses in C. gloeosporioides, causing Stylosanthes anthracnose. In Colletotrichum higginsianum, the extracellular LysM effector proteins play dual roles in appressorial function and suppression of chitin-triggered plant immunity [6]. The fungalysin metalloprotease is specifically expressed during the biotrophic stage of infection and is involved in the development of infected mycelium in Colletotrichum graminicola [7]. The effector, CtNUDIX, can disrupt plant cell 
plasma membrane integrity and cell death, leading to a switch of lifestyle from biotrophic mode to necrotrophy in Colletotrichum truncatum [8].

Most plant pathogenic fungi differentiate infection structures, named appressoria, to penetrate the cuticle and cell wall of their hosts; after successful penetration, other specialized structures, called primary hyphae, develop and differentiate into biotrophic invasive hyphae. Previous work suggested that appressoria, primary hyphae, and invasive hyphae were the main structures for effector protein secretion in Magnaporthe oryzae [9]. Among these effector proteins, four proteins, named MoBAS1-4, were confirmed to be fungal biotrophy-associated secreted proteins in M. oryzae [10]. Transcriptional analysis showed that MoBAS were all upregulated in invasive hyphae. Protein sequence analysis revealed that MoBAS2, MoBAS3, and MoBAS4 encode secreted proteins with conserved Cys residues. In the genome of $C$. gloeosporioides from $H$. brasiliensis, the gene BAS2 encoding homologous protein to MoBAS2 was identified. In our previous work, deletion of part of the coding sequence of BAS2 led to decreased pathogenicity of $C$. gloeosporioides, but the mechanism of BAS2 in regulating the virulence of the pathogen remains unclear; besides, whether BAS2 is involved in vegetative growth and conidiation is still unknown. Therefore, in the present study, the coding sequence of BAS2 was completely deleted from the C. gloeosporioides genome, and the functions of BAS2 in regulating growth and pathogenicity and the possible mechanism were investigated.

\section{Results}

\subsection{Generation of the $\triangle B A S 2$ Mutant}

In our previous work, knocking out part of the BAS2 coding sequence led to decreased pathogenicity of $C$. gloeosporioides to $H$. brasiliensis leaves [11]. To fully understand the functions of BAS2, the nucleotide sequence of BAS2 was fully deleted from the genome of $C$. gloeosporioides using the homologous recombination strategy (Supplementary Figure S3A). After the transformation, the Hygromycin-resistant colonies were analyzed with PCR to ensure correct integration into the gene locus. As shown in Supplementary Figure S3A, primer pairs with one primer outside of the flanking region and one inside the replacement fragments were used. The results show that the replacement fragments were correctly integrated into the BAS2 locus in the mutants. The knockout mutants were named $\triangle \mathrm{BAS} 2$. Then the transformants were purified by single conidia isolation to exclude heterokaryons.

\subsection{BAS2 Is Involved in Vegetative Growth and Conidiation}

As shown in Figure 1A, knocking out of BAS2 led to mild inhibition of vegetative growth in vitro. After being cultured in liquid complete medium for 3 and 4 days, the concentrations of conidia of the wild-type (WT) strain were up to $2.8 \times 10^{6}$ and $9.1 \times 10^{6}$ conidia $/ \mathrm{mL}$, while those of $\triangle \mathrm{BAS} 2$ were only $1.0 \times 10^{6}$ and $3.5 \times 10^{6}$ conidia/mL (Figure $1 \mathrm{~B}$ ), suggesting significant impairment of conidia generation of the mutant. 

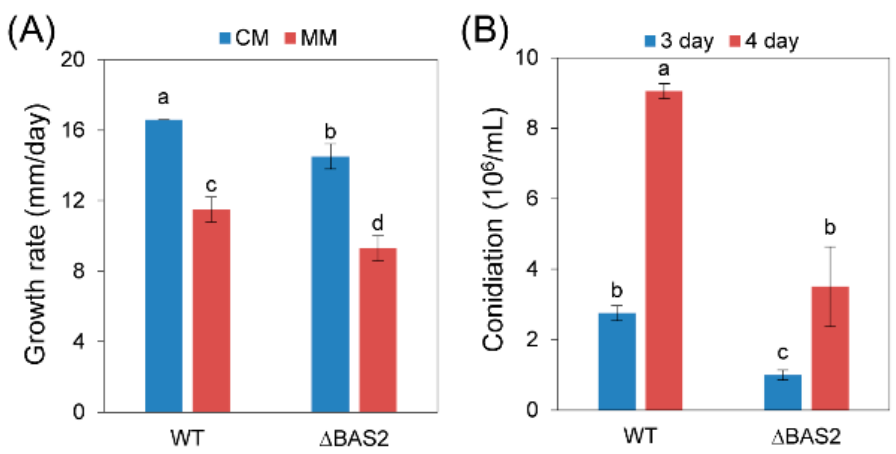

Figure 1. Growth rate and conidiation assays. (A) Growth rate of wild-type (WT) and $\triangle B A S 2$ cultured on complete medium (CM) and minimal medium (MM); (B) conidiation of WT and $\triangle \mathrm{BAS} 2$. Bars represent standard deviation (SD). Columns with different letters indicate significant difference $(p<0.05)$.

\subsection{BAS2 Are Required for Pathogenicity}

To test whether pathogenicity and penetration ability were impaired in $\triangle \mathrm{BAS} 2$, the pathogens were inoculated on leaves that were prewounded or nonwounded. The results show that when inoculated on prewounded leaves, both WT and $\triangle \mathrm{BAS} 2$ showed $100 \%$ incidence of disease (Figure $2 \mathrm{~B}$ ), and the lesions caused by $\triangle \mathrm{BAS} 2$ and by WT had nearly the same diameter (Figure 2C). When inoculated on nonwounded leaves, the incidence of disease of $\triangle \mathrm{BAS} 2$ decreased to $23 \%$, while that of WT was still up to $77 \%$ (Figure 2E); furthermore, the lesions caused by $\triangle \mathrm{BAS} 2$ were about half the diameter of those caused by WT (Figure 2F).
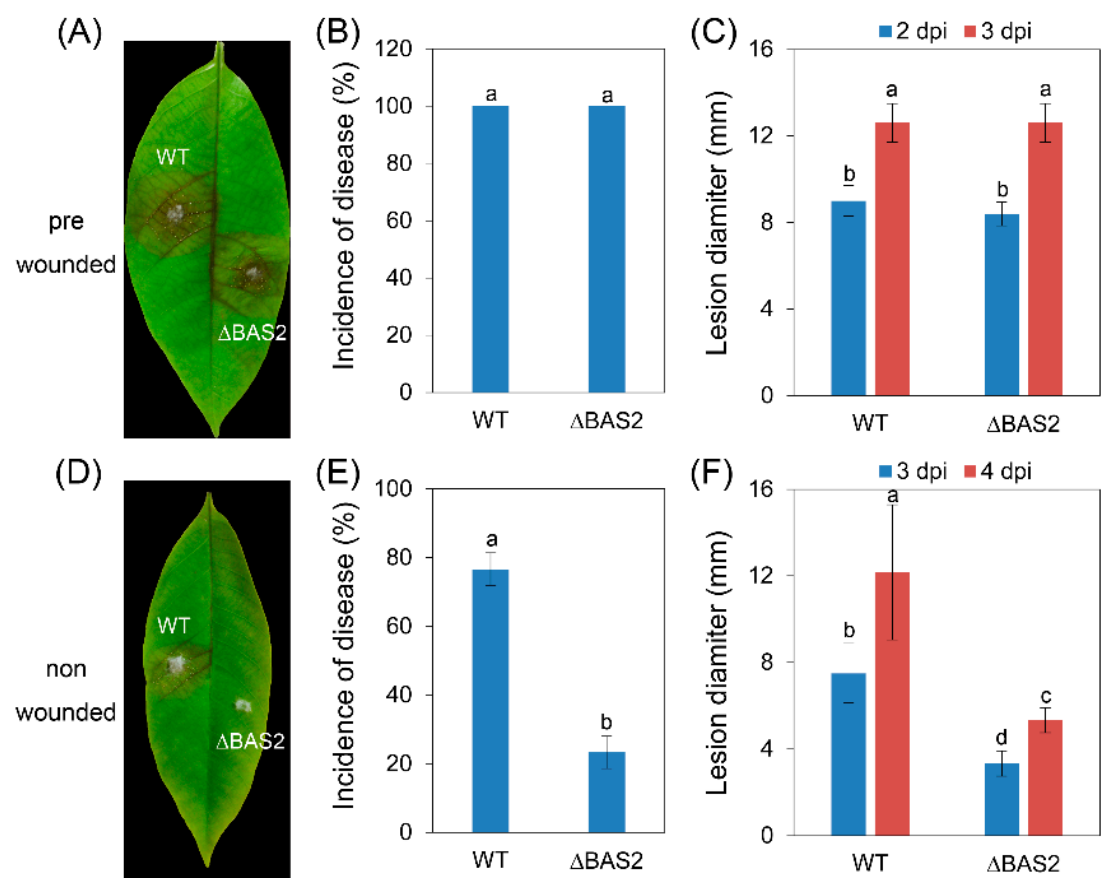

Figure 2. Pathogenicity assay on rubber-tree leaves. (A,D) Disease symptoms of rubber-tree leaves at 3 days post-inoculation (dpi). (B,E) Mean incidence of disease of rubber-tree leaves at 3 dpi. (C,F) Mean lesion diameters after at different dpi. (A-C) Rubber-tree leaves were prewounded before the inoculation. (D-F) Rubber-tree leaves were nonwounded before the inoculation. Bars represent standard deviation (SD). Columns with different letters indicate significant difference $(p<0.05)$. 


\subsection{Subcellular Localization of BAS2-GFP in C. gloeosporioides}

In the present study, the nitrate reductase (niaD) gene locus was used to construct the BAS2-green fluorescent protein (GFP) fusion expressing transformants with the homologous recombination strategy (Supplementary Figure S4). The preliminary experiments showed that niaD is not required for vegetative growth or full virulence in C. gloeosporioides (Supplementary Figure S5), and similar phenotypes were found in the BcniaD deletion mutant of Botrytis cinerea [12]. Hence, the vector pCgNDHTN was suitable for the reporter gene constructs. The BAS2-GFP expressing transformant was constructed using $\mathrm{pCgNDHTN}$. The transformants expressing only GFP showed strong fluorescence all through the cytoplasm in C. gloeosporioides. In contrast, the transformants expressing the BAS2-GFP fusion protein only showed weak fluorescence in conidia; furthermore, no fluorescence was observed in the hyphae (Figure 3), suggesting that BAS2 was secreted out of the cell.
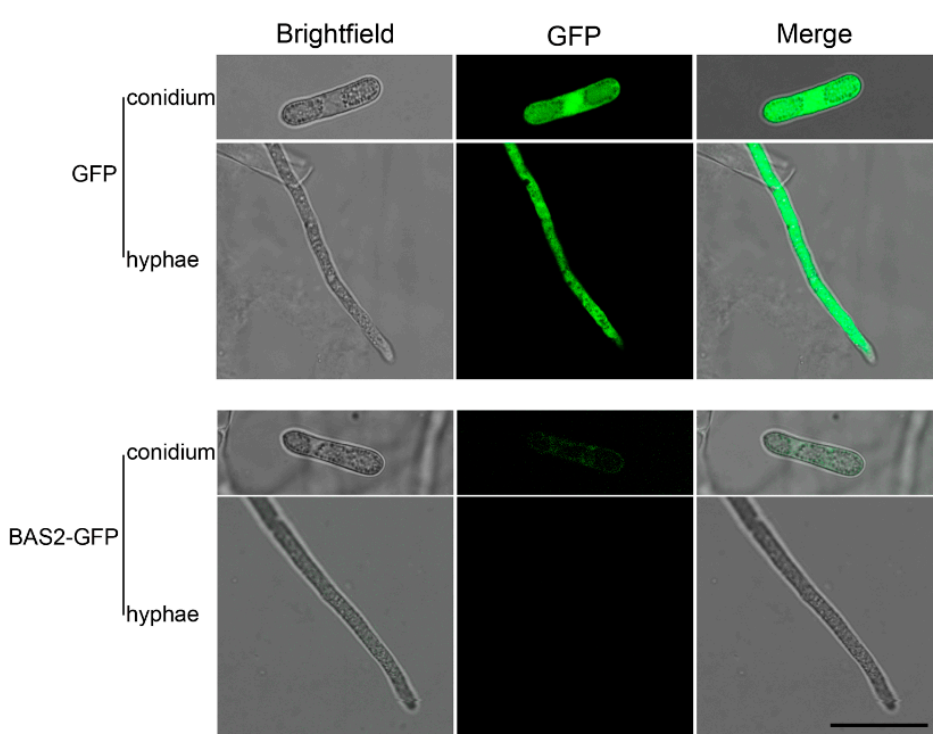

Figure 3. Subcellular localization of BAS2-green fluorescent protein (GFP) in conidia and hyphae of C. gloeosporioides. Scale bar $=20 \mu \mathrm{m}$.

\subsection{Subcellular Localization of BAS2-GFP in Mesophyll Cell Protoplasts of H. brasiliensis}

Transient expression of BAS2-GFP in mesophyll cell protoplasts of $H$. brasiliensis was conducted to detect the subcellular localization of BAS2-GFP in host cells. As shown in Figure 4, fluorescence of BAS2-GFP was observed all through the cytoplasm of the protoplasts of $\mathrm{H}$. brasiliensis, showing the same pattern as the control that expressed empty GFP, suggesting that BAS2 was localized in cytoplasm in the host cell. 


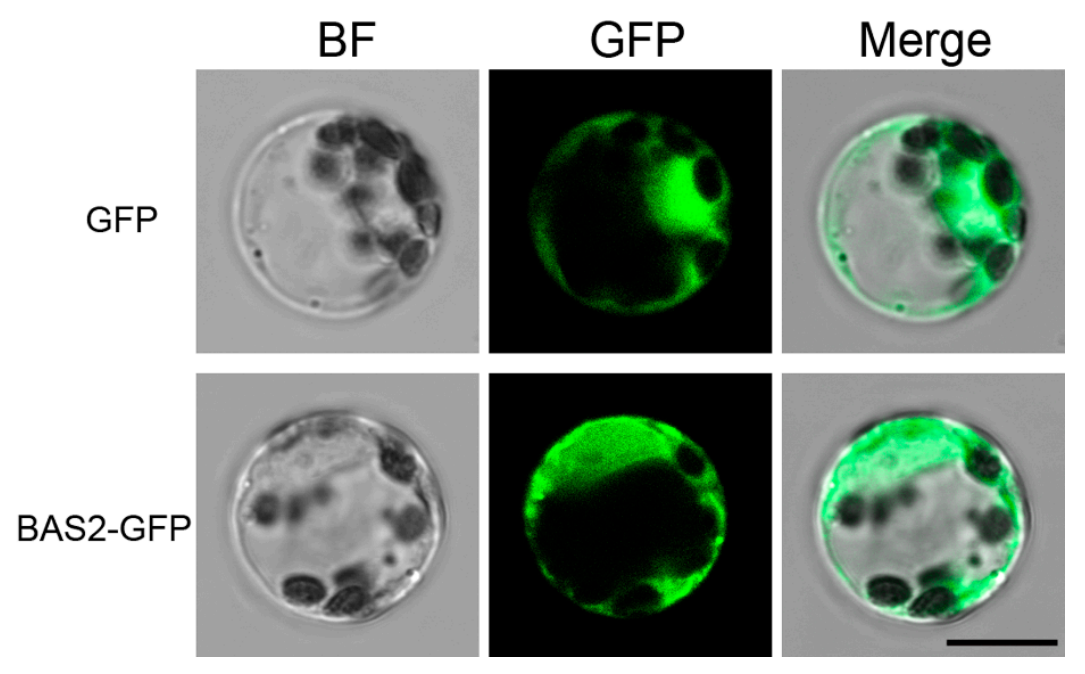

Figure 4. Subcellular localization of BAS2-GFP in mesophyll cell protoplasts of $H$. brasiliensis. Scale bar $=10 \mu \mathrm{m}$.

\subsection{BAS2 Is Involved in Extracellular Protein Secretion}

To investigate whether BAS2 was involved in the protein secretion process, comparative extracellular proteomics analysis was conducted. Two-dimensional (2D) gel electrophoresis was conducted, and a total of 420 protein spots were detected on the Coomassie brilliant blue stained 2D gels, including samples from both WT and $\triangle \mathrm{BAS2}$. Quantitative image analysis revealed that 21 protein spots were downregulated (Figure 5) and 51 were upregulated (Supplementary Figure S6) in abundance in $\triangle B A S 2$. The 22 downregulated protein spots were excised and submitted to tandem mass spectrometry and identified by database searching with the Mascot search engine (Table 1). The identified proteins were classified into six functional categories according to their biological function: cell structure, redox, metabolism, secondary metabolism, cell wall degradation, and unknown.

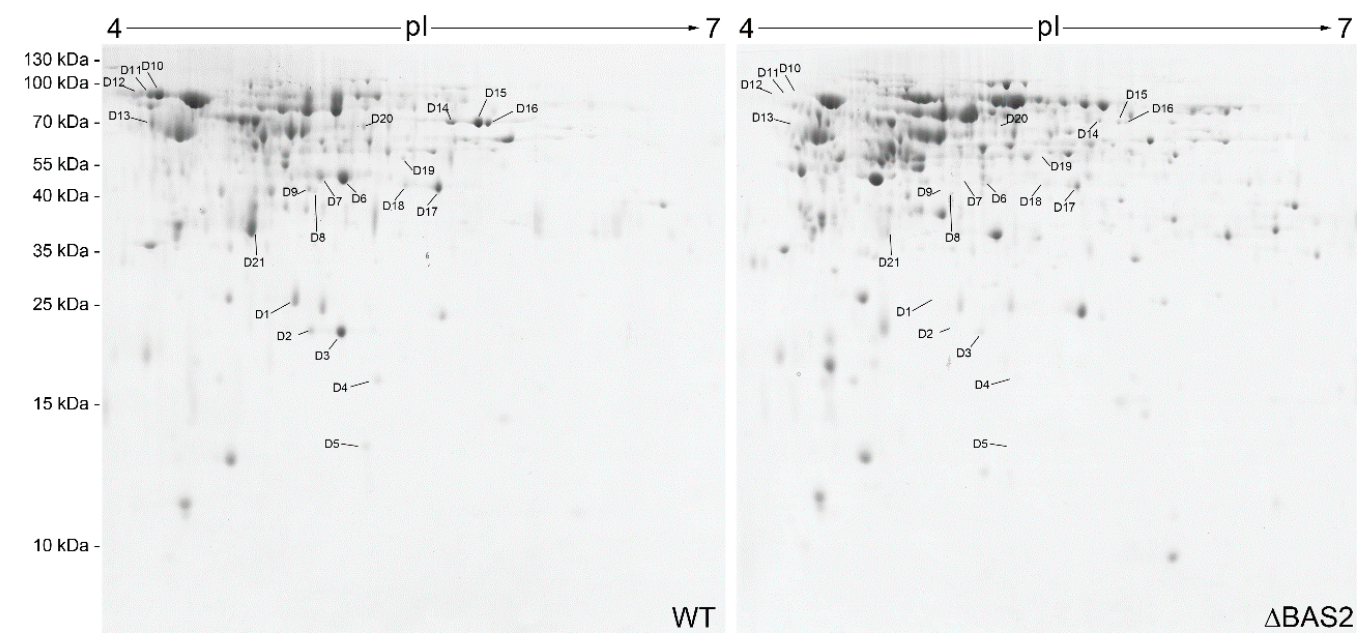

Figure 5. Two-dimensional patterns of extracellular proteomes of WT and $\triangle \mathrm{BAS} 2$. Arrows indicate protein spots that were downregulated in abundance more than 1.5-fold between WT and $\triangle \mathrm{BAS} 2$. Numbering of protein spots corresponds to numbering in Table 1. 
Table 1. Proteins identified by quadrupole-time-of-flight tandem mass spectrometry. The accession numbers of the proteins of $C$. gloeosporioides Nara gc5, which showed the most homology to the protein spots, are listed. NP, number of matched peptides; SC, amino acid sequence coverage for identified proteins; WT vs. $\triangle \mathrm{BAS2}$, average fold change of relative abundance of specific spot of WT versus $\triangle \mathrm{BAS} 2$ from three biological repeats; $\infty$, corresponding spot appeared in WT but not in $\triangle \mathrm{BAS} 2$.

\begin{tabular}{|c|c|c|c|c|c|c|}
\hline Spot & Protein Function & Accession Number & NP & SC (\%) & WT vs. $\triangle$ BAS2 & Biological Function \\
\hline \multicolumn{7}{|c|}{ Cell structure } \\
\hline D1 & Actin & ELA34037.1 & 2 & 6 & $\infty$ & Cytoskeleton \\
\hline \multicolumn{7}{|c|}{ Redox } \\
\hline D6 & Peroxidase & ELA30823.1 & 14 & 37 & 38.56 & Redox \\
\hline D8 & Peroxidase & ELA30823.1 & 11 & 30 & $\infty$ & Redox \\
\hline D9 & Peroxidase & ELA30823.1 & 12 & 34 & $\infty$ & Redox \\
\hline \multicolumn{7}{|c|}{ Metabolism } \\
\hline D7 & $\begin{array}{l}\text { Endonuclease/exonuclease/phosphatase } \\
\text { family protein }\end{array}$ & ELA30823.1 & 13 & 35 & $\infty$ & Metabolism \\
\hline D11 & Choline dehydrogenase & ELA24295.1 & 10 & 17 & $\infty$ & Choline metabolism \\
\hline D12 & Choline dehydrogenase & ELA24295.1 & 9 & 16 & $\infty$ & Choline metabolism \\
\hline D13 & $\begin{array}{l}\text { Flavine adenine dinucleotide-dependent } \\
\text { oxygenase }\end{array}$ & ELA25647.1 & 10 & 22 & 33.04 & Metabolism \\
\hline D15 & $\begin{array}{l}\text { Flavine adenine dinucleotide-binding } \\
\text { dehydrogenase }\end{array}$ & ELA24979.1 & 3 & 5 & 13.20 & Metabolism \\
\hline D21 & Enolase & ELA34631.1 & 15 & 30 & 14.04 & Glycolytic pathways \\
\hline \multicolumn{7}{|c|}{ Secondary metabolism } \\
\hline D10 & Indoleacetamide hydrolase (IaaH) & ELA36057.1 & 8 & 14 & $\infty$ & IAA synthesis \\
\hline D14 & Versicolorin b synthase & ELA30672.1 & 13 & 19 & 26.33 & Aflatoxin synthesis \\
\hline D16 & Versicolorin b synthase & ELA30672.1 & 16 & 25 & 45.11 & Aflatoxin synthesis \\
\hline D19 & Tyrosinase central domain containing protein & ELA33276.1 & 4 & 8 & $\infty$ & Melanin synthesis \\
\hline \multicolumn{7}{|c|}{ Cell wall degradation } \\
\hline D17 & Endo- $\beta-1,6$-glucanase & ELA27535.1 & 11 & 21 & 1.70 & Degradation of chitin \\
\hline D18 & Endo- $\beta-1,6$-glucanase & ELA27535.1 & 8 & 19 & $\infty$ & Degradation of chitin \\
\hline D20 & Exopolygalacturonase & ELA28364.1 & 4 & 9 & 2.29 & Degradation of pectin \\
\hline \multicolumn{7}{|c|}{ Unknown } \\
\hline D2 & Not identified & - & - & - & $\infty$ & - \\
\hline D3 & Not identified & - & - & - & 14.69 & - \\
\hline D4 & Not identified & - & - & - & $\infty$ & - \\
\hline D5 & Not identified & - & - & - & $\infty$ & - \\
\hline
\end{tabular}

\section{Discussion}

In recent years, an increasing number of effectors have been identified in fungal pathogens. Four Cys-rich secreted proteins related to biotrophy were identified and named biotrophy-associated secreted (BAS) proteins in M. oryzae [10]. By searching the genome, one gene-encoding protein homologous to MoBAS2 in C. gloeosporioides from H. brasiliensis was identified and named BAS2. Sequence alignment of the predicted BAS2 proteins of C. gloeosporioides from H. brasiliensis, Colletotrichum gloeosporioides Nara gc5, C. higginsianum, C. graminicola, M. oryzae, and Podospora anserine revealed that BAS2 proteins are well conserved in filamentous fungi (Supplementary Figures S1 and S2).

For functional analysis, knockout mutants of BAS2 were constructed (Supplementary Figure S3). The growth and conidiation of $\triangle \mathrm{BAS} 2$ were analyzed in vitro. The results show that only a very slight decrease in growth rate was observed in $\triangle \mathrm{BAS} 2$ compared with WT, but conidia generation of $\triangle B A S 2$ was reduced about three times compared with WT; these results reveal that BAS2 is not necessary for vegetative growth but is important for normal asexual reproduction in C. gloeosporioides. Successful penetration into the host tissue is the first step of pathogenicity. To test whether BAS2 is involved in the penetration process, C. gloeosporioides strains were inoculated on leaves in two ways, with leaves prewounded or nonwounded. The results show that when inoculated on the wounds of leaves, both WT and $\triangle$ BAS2 caused 100\% incidence of disease, but when inoculated on nonwounded leaves, the incidence of $\triangle B A S 2$-caused disease decreased sharply compared with WT, suggesting that BAS2 is important for the penetration process of pathogen to host. Besides, the diameters of lesions caused by $\triangle B A S 2$ were nearly the same as those caused by WT when inoculated on prewounded leaves, suggesting that BAS2 is not required for mycelial growth in plant tissue. When inoculated on nonwounded leaves, the diameters of lesions caused by $\triangle$ BAS2 were smaller than those caused by 
WT, but this might be due to decreased penetration ability of $\triangle \mathrm{BAS2}$. However, deletion of MoBAS in M. oryzae did not show a change in mycelial growth, conidiation, or pathogenicity [10].

Subcellular localization analysis showed that MoBAS2 localized to the biotrophic interfacial complex (BIC) of invasive hyphae in M. oryzae and had secretion characteristics [10,13]. In the present study, transformants overexpressing BAS2-GFP fusion protein were constructed. Microscopic analysis showed that little fluorescence was observed in the hyphae of the transformants, suggesting that BAS2 functions as a secreted protein in C. gloeosporioides from H. brasiliensis. After being secreted into the host cell, effector proteins could function as cytoplasmic proteins, bind to the plasma membrane proteins [14], and even transfer into the nuclei [15]. To investigate the subcellular localization of BAS2 in the host cells, transient expression of BAS2-GFP in the protoplasts of $H$. brasiliensis was conducted, and the results show that BAS2 was localized in cytoplasm in H. brasiliensis cells.

Effector proteins could not only directly bind to the targets in the host cell to disturb the host immune system, but also act as regulatory proteins to control the physical activities of pathogens themselves [16]. In the present study, BAS2 was involved in the penetration process of $C$. gloeosporioides from $H$. brasiliensis, suggesting its close relation to the protein secretion process. Therefore, the extracellular proteome of $\triangle \mathrm{BAS} 2$ was analyzed. The results show that a total of 21 protein spots representing 12 proteins were significantly downregulated in the 2D gel images of $\triangle \mathrm{BAS} 2$ (Figure 5). Interestingly, another 51 protein spots were upregulated in $\triangle \mathrm{BAS} 2$ (Supplementary Figure S6). Since deletion of BAS2 led to decreased conidiation and pathogenicity, only the downregulated protein spots were investigated in subsequent analysis. Among the 12 proteins, 2 enzymes involved in cell wall degradation were downregulated in the mutant. Colletotrichum species use a combination of mechanical force and enzymatic degradation to penetrate into the host surfaces. Therefore, a reduced abundance of the two enzymes might be the cause of decreased disease incidence in $\triangle \mathrm{BAS} 2$ (Figure 2E). Oxidative burst is one of the earliest events in the plant's hypersensitive response to pathogen attack. In the previous work, we found that inoculation of C. gloeosporioides could cause a significant increase of hydrogen peroxide accumulation in H. brasiliensis leaves [17]. The proteome analysis here showed that C. gloeosporioides secreted abundant peroxidase to the extracellular spaces, suggesting that peroxidase plays an important role in regulating the redox level of the host tissue. In $\triangle \mathrm{BAS} 2$, the abundance of extracellular peroxidase decreased dramatically, which could lead to decreased disease incidence and lesion development (Figure 2E,F). It is well known that plant pathogens could secret auxin to facilitate pathogenicity to host plants $[18,19]$. Here we found that indoleacetamide hydrolase (IaaH), one of the key enzymes for auxin synthesis in fungi, was secreted to the host cell by C. gloeosporioides. However, deletion of BAS2 caused a dramatic decrease in the abundance of the enzyme, which might be another cause of the decreased pathogenicity in $\triangle \mathrm{BAS} 2$. Melanin is a secondary metabolite in fungi, which plays important roles in conferring tolerance to environmental stresses, differentiating conidia and appressoria, and pathogenicity to the host [20,21]. In $\triangle$ BAS2, tyrosinase, one of the key enzymes in the biosynthesis of melanin, was downregulated significantly. Besides, the protein abundance of versicolorin b synthase, which is involved in aflatoxin biosynthesis, was also decreased in $\triangle B A S 2$. Actin, a component of intracellular cytoskeleton, was also identified from the extracellular proteome of $C$. gloeosporioides, suggesting that there might be autophagic fungal cell death during the infection process [22]. However, the actin protein was not observed in $\triangle \mathrm{BAS} 2$, indicating decreased infection ability of the mutant. Moreover, five identified proteins involved in metabolism were also downregulated in $\triangle \mathrm{BAS} 2$, suggesting that the pathogen could also interfere with the normal metabolism of the host cell. The previous studies revealed that $C$. graminicola gene expression is highly dynamic and is induced in successive waves during specific stages of the infection process [4].

Taken together, BAS2 is involved in the secretion of proteins closely related to pathogenicity and plays an important role in conidiation and pathogenicity of C. gloeosporioides from H. brasiliensis. 


\section{Materials and Methods}

\subsection{Fungal Strains and Culture Conditions}

C. gloeosporioides strain was isolated from $H$. brasiliensis leaves with anthracnose and grown on potato dextrose agar (PDA) at $28^{\circ} \mathrm{C}$.

\subsection{Construction of the Knockout Vector of BAS2}

To construct the knockout vector of BAS2, plasmid pBluescript II KS (+) (pBS) was used as the backbone. First, the Hygromycin phosphotransferase gene expressing cassette (HPH) was amplified from the vector $\mathrm{pKoV} 21$ and ligated into $\mathrm{pBSat}$ of the XbaI/EcoRI sites to construct the vector $\mathrm{pBS}-H P H$. Then the $5^{\prime}$ and $3^{\prime}$ flanking regions of the BAS2 gene were amplified from genomic DNA with the restriction sites added at both ends of flank, as shown in Supplementary Figure S3A. After that, the 2 flanking regions were ligated into the vector $\mathrm{pBS}-\mathrm{HPH}$ at the corresponding sites to yield the knockout vector. The knockout vector was linearized with SacI (Thermo Fisher Scientific, Waltham, MA, USA) before transformation of the C. gloeosporioides strain. All the primers used are listed in Supplementary Table S1.

\subsection{Transformation of C. gloeosporioides, PCR Diagnosis, and Single Conidia Purification}

Protoplast formation and transformation of C. gloeosporioides were performed using the methods described by Wang et al. [17]. The Hygromycin-resistant strains were isolated and analyzed by PCR with the primer pairs shown in Supplementary Figure S3, which are diagnostic for homologous integration of the $5^{\prime}$ part and $3^{\prime}$ part. Then the correct transformants were purified by single conidia isolations.

\subsection{Growth and Conidiation Assay}

The C. gloeosporioides strains were inoculated on complete medium and minimal medium [23] and grown for 5 days. The diameters of the colonies were recorded and the growth rates were calculated. Conidia were harvested from C. gloeosporioides strains grown on PDA medium, washed with dd $\mathrm{H}_{2} \mathrm{O}$, and inoculated into $50 \mathrm{~mL}$ liquid complete medium, making an initial concentration of $10^{3} \mathrm{conidia} / \mathrm{mL}$. Then the strains were cultured at $28{ }^{\circ} \mathrm{C}$ at $150 \mathrm{rpm}$ for the desired time, and the conidia number was calculated under a microscope.

\subsection{Pathogenicity Assay}

The C. gloeosporioides strains were grown on PDA medium for 2 days, and pieces of mycelia together with agar (about $1 \mathrm{~mm}^{3}$ ) were excised from the growing edge of the colonies. The H. brasiliensis leaves at the "light green" stage were detached from rubber tree Varity 73-3-97, and half of the leaves were wounded with sterilized needles. For the pathogenicity assay, mycelia were inoculated on the leaves that were prewounded or nonwounded. After that, the inoculated leaves were kept in a moist chamber at $28^{\circ} \mathrm{C}$ under natural illumination for 4 days and the disease symptoms were scored. Each treatment contained 3 replicates of 10 leaves, and the entire experiment was repeated twice.

\subsection{Generation of BAS2-GFP Fusion Expressing Mutant}

To construct an efficient expression system for C. gloeosporioides, the vector pCgNDHTN was constructed according to Schumacher [12]. Briefly, the gene locus of niaD was used for targeted integration of reporter gene constructs together with promoter and terminator. The strategy for the vector construction is shown in Supplementary Figure S4A. First, the $5^{\prime}$ and $3^{\prime}$ flanking regions of the niaD were amplified and ligated into the vector $\mathrm{pBS}$; second, the $H P H$ cassette was amplified and ligated into the vector $\mathrm{pBS}$; finally, the promoter of ToxA and the terminator of nos were amplified from the vector $\mathrm{pCT74}$ [24] and ligated into the vector $\mathrm{pBS}$ to generate $\mathrm{pCgNDHTN}$. 
Green fluorescent protein (GFP) was used as the reporter gene to analyze the subcellular location of BAS2 in C. gloeosporioides. To construct the BAS2-GFP fusion vector, the open reading frame of BAS2 and the coding sequence of GFP with an N-terminal linker [12] were amplified and ligated into the PstI/SpeI and SpeI/XbaI sites of pCgNDHTN, respectively. The BAS2-GFP fusion vector was then named pCgNDHTN-BAS2-GFP (Supplementary Figure S4B), and the vector was linearized with SacI before transformation of $C$. gloeosporioides. The transformants were analyzed by PCR to ensure correct integration into the niaD gene locus. Then the correct mutants expressing BAS2-GFP were grown in the liquid complete medium, and the subcellular localization of BAS2-GFP fusion protein in conidia and hyphae of C. gloeosporioides was observed under a Leica TCS SP8 laser scanning confocal microscope, with argon laser excitation of $488 \mathrm{~nm}$ and an emission wavelength range of 505-525 nm. The intensity of the argon laser in laser configuration and intensity of laser line 488 in the acquire section were set to $20 \%$ and $15 \%$, respectively. Pinhole was set to 1.8 Airy units. To compare the fluorescence intensity of GFP in samples, all optical sections were acquired under identical conditions.

\subsection{Construction of BAS2-GFP Fusion Expressing Vector and Transient Expression in Mesophyll Cell Protoplasts of $\mathrm{H}$. brasiliensis}

The BAS2-GFP fusion expressing vector was constructed by ligating the open reading frame of BAS2 and the GFP sequence into the downstream of the cauliflower mosaic virus 35S promoter. The rubber-tree mesophyll cell protoplasts were prepared and transformed according to Zhang et al. [25]. The subcellular localization of BAS2-GFP fusion protein in mesophyll cell protoplasts of $H$. brasiliensis was observed under the confocal, with the protoplasts expressing GFP alone as the empty control.

\subsection{Extracellular Proteomic Analysis}

To induce the expression of extracellular proteins, modified Czapeck liquid medium in which glucose was replaced with apple pectin was used to culture $C$. gloeosporioides. C. gloeosporioides strains were grown for 7 days on PDA medium, then the conidia were collected, washed 2 times with $\mathrm{ddH}_{2} \mathrm{O}$, and inoculated into $200 \mathrm{~mL}$ Czapeck liquid medium to make an initial concentration of $10^{3}$ conidia $/ \mathrm{mL}$. After being cultured for 3 days at $28^{\circ} \mathrm{C}$ at $160 \mathrm{rpm}$, the supernatant was filtrated and centrifuged to remove impurities. The extracellular proteins were precipitated from the supernatant by adding sodium deoxycholate (Sigma-Aldrich) to the final concentration of $0.03 \%(w / v)$, followed by incubation on ice for $30 \mathrm{~min}$; subsequently, trichloric acetic acid (100\%) (Sigma-Aldrich, St. Louis, MO, USA) was added to the mixture to a final concentration of $10 \%(v / v)$, followed by incubation on ice for another $30 \mathrm{~min}$. Then the precipitated proteins were collected by centrifugation at $16,000 \times g$ at $4{ }^{\circ} \mathrm{C}$ for $30 \mathrm{~min}$ and washed 3 times with cold acetone. To remove residual apple pectin, the air-dried precipitate was redissolved in extraction buffer (0.5 M Tris- $\mathrm{HCl}, \mathrm{pH} 8.3,2 \%(v / v) \mathrm{NP}-40,20 \mathrm{mM} \mathrm{MgCl} 2,2 \%(v / v)$ b-mercaptoethanol, and $1 \mathrm{mM}$ Phenylmethanesulfonyl fluoride), and an equal volume of Tris- $\mathrm{HCl}$ ( $\mathrm{pH}$ 7.8) buffered phenol was added into the mix to extract proteins. After centrifugation, proteins were precipitated from the final phenol phase with $5 \mathrm{vol}$ of ice-cold saturated ammonium acetate in methanol overnight at $-20^{\circ} \mathrm{C}$. Then the proteins were collected by centrifugation and washed twice with cold saturated ammonium acetate in methanol and acetone. The air-dried proteins were solubilized in the thiourea/urea lysis buffer, and 2D gel electrophoresis and MALDI-TOF/TOF mass spectrometer analyses were conducted according to Wang et al. [17].

Supplementary Materials: Supplementary materials can be found at http:/ /www.mdpi.com/1422-0067/19/7/ $1860 /$ s1.

Author Contributions: B.A. and C.H. conceived and designed the research. B.A., W.W., and Y.G. performed the experiments. Q.W. performed the confocal assays. B.A. and H.L. analyzed the data. All authors contributed to writing this manuscript and approved its final version.

Funding: This study was funded by the National Natural Science Foundation of China (31560044 and 31571967).

Conflicts of Interest: The authors declare no conflict of interest. 


\section{References}

1. Koeck, M.; Hardham, A.R.; Dodds, P.N. The role of effectors of biotrophic and hemibiotrophic fungi in infection. Cell. Microbiol. 2011, 13, 1849-1857. [CrossRef] [PubMed]

2. Göhre, V.; Robatzek, S. Breaking the barriers: Microbial effector molecules subvert plant immunity. Annu. Rev. Phytopathol. 2008, 46, 189-215. [CrossRef] [PubMed]

3. Rafiqi, M.; Ellis, J.G.; Ludowici, V.A.; Hardham, A.R.; Dodds, P.N. Challenges and progress towards understanding the role of effectors in plant-fungal interactions. Curr. Opin. Plant Biol. 2012, 15, 477-482. [CrossRef] [PubMed]

4. O'Connell, R.J.; Thon, M.R.; Hacquard, S.; Amyotte, S.G.; Kleemann, J.; Torres, M.F.; Damm, U.; Buiate, E.A.; Epstein, L.; Alkan, N.; et al. Lifestyle transitions in plant pathogenic Colletotrichum fungi deciphered by genome and transcriptome analyses. Nat. Genet. 2012, 44, 1060-1065. [CrossRef] [PubMed]

5. Stephenson, S.A.; Hatfield, J.; Rusu, A.G.; Maclean, D.J.; Manners, J.M. CgDN3: An essential pathogenicity gene of Colletotrichum gloeosporioides necessary to avert a hypersensitive-like response in the host Stylosanthes guianensis. Mol. Plant Microbe Interact. 2000, 13, 929-941. [CrossRef] [PubMed]

6. Takahara, H.; Hacquard, S.; Kombrink, A.; Hughes, H.B.; Halder, V.; Robin, G.P.; Hiruma, K.; Neumann, U.; Shinya, T.; Kombrink, E.; et al. Colletotrichum higginsianum extracellular lysm proteins play dual roles in appressorial function and suppression of chitin-triggered plant immunity. New Phytol. 2016, 211, 1323-1337. [CrossRef] [PubMed]

7. Sanzmartín, J.M.; Pachecoarjona, J.R.; Bellorico, V.; Vargas, W.A.; Monod, M.; Díazmínguez, J.M.; Thon, M.R.; Sukno, S.A. A highly conserved metalloprotease effector enhances virulence in the maize anthracnose fungus Colletotrichum graminicola. Mol. Plant Pathol. 2015, 17, 1048-1062. [CrossRef] [PubMed]

8. Bhadauria, V.; Banniza, S.; Vandenberg, A.; Selvaraj, G.; Wei, Y. Overexpression of a novel biotrophy-specific Colletotrichum truncatum effector, CtNUDIX, in hemibiotrophic fungal phytopathogens causes incompatibility with their host plants. Eukaryot. Cell 2013, 12, 2-11. [CrossRef] [PubMed]

9. Kleemann, J.; Rincon-Rivera, L.J.; Takahara, H.; Neumann, U.; Ver Loren van Themaat, E.H.; van der Does, C.; Hacquard, S.; Stüber, K.; Will, I.; Schmalenbach, W.; et al. Sequential delivery of host-Induced virulence effectors by appressoria and intracellular hyphae of the phytopathogen Colletotrichum higginsianum. PLoS Pathog. 2012, 8, e1002643. [CrossRef]

10. Mosquera, G.; Giraldo, M.C.; Khang, C.H.; Coughlan, S.; Valent, B. Interaction transcriptome analysis identifies Magnaporthe oryzae BAS1-4 as biotrophy-associated secreted proteins in rice blast disease. Plant Cell 2009, 21, 1273-1290. [CrossRef] [PubMed]

11. Wang, Q.; He, C.; Luo, H. Pathogenicity analysis and construction of CgBASP2 knockout mutant of Colletotrichum gloeosporioides infecting Hevea brasiliensis. J. Trop. Biol. 2015, 6, 41-46. (In Chinese)

12. Schumacher, J. Tools for Botrytis cinerea: New expression vectors make the gray mold fungus more accessible to cell biology approaches. Fungal Genet. Biol. 2012, 49, 483-497. [CrossRef] [PubMed]

13. Khang, C.H.; Berruyer, R.; Giraldo, M.C.; Kankanala, P.; Park, S.Y.; Czymmek, K.; Kang, S.; Valent, B. Translocation of Magnaporthe oryzae effectors into rice cells and their subsequent cell-to-cell movement. Plant Cell 2010, 22, 1388-1403. [CrossRef] [PubMed]

14. Xin, X.F.; Nomura, K.; Ding, X.; Chen, X.; Wang, K.; Aung, K.; Uribe, F.; Rosa, B.; Yao, J.; Chen, J.; et al. Pseudomonas syringae effector avirulence protein $\mathrm{E}$ localizes to the host plasma membrane and down-regulates the expression of the nonrace-specific disease resistance1/harpin-induced1-like13 gene required for antibacterial immunity in Arabidopsis. Plant Physiol. 2015, 169, 793-802. [CrossRef] [PubMed]

15. Hewezi, T.; Juvale, P.S.; Piya, S.; Maier, T.R.; Rambani, A.; Rice, J.H.; Mitchum, M.G.; Davis, E.L.; Hussey, R.S.; Baum, T.J. The cyst nematode effector protein 10a07 targets and recruits host posttranslational machinery to mediate its nuclear trafficking and to promote parasitism in Arabidopsis. Plant Cell 2015, 27, 891-907. [CrossRef] [PubMed]

16. Marshall, R.; Kombrink, A.; Motteram, J.; Lozareyes, E.; Lucas, J.; Hammond-Kosack, K.E.; Thomma, B.P.; Rudd, J.J. Analysis of two in planta expressed LysM effector homologs from the fungus Mycosphaerella graminicola reveals novel functional properties and varying contributions to virulence on wheat. Plant Physiol. 2011, 156, 756-769. [CrossRef] [PubMed] 
17. Wang, Q.; An, B.; Hou, X.; Guo, Y.; Luo, H.; He, C. Dicer-like proteins regulate the growth, conidiation, and pathogenicity of Colletotrichum gloeosporioides from Hevea brasiliensis. Front. Microbiol. 2018, 8, 2621. [CrossRef] [PubMed]

18. Reineke, G.; Heinze, B.; Schirawski, J.; Buettner, H.; Kahmann, R.; Basse, C.W. Indole-3-acetic acid (IAA) biosynthesis in the smut fungus Ustilago maydis, and its relevance for increased IAA levels in infected tissue and host tumour formation. Mol. Plant Pathol. 2008, 9, 339-355. [CrossRef] [PubMed]

19. Yin, C.; Park, J.J.; Gang, D.R.; Hulbert, S.H. Characterization of a tryptophan 2-monooxygenase gene from Puccinia graminis f. sp. tritici involved in auxin biosynthesis and rust pathogenicity. Mol. Plant Microbe Interact. 2014, 27, 227-235. [CrossRef] [PubMed]

20. Chumley, F.G.; Valent, B. Genetic analysis of melanin-deficient, nonpathogenic mutants of Magnaporthe grisea. Mol. Plant Microbe Interact. 1990, 3, 135-143. [CrossRef]

21. Kawamura, C.; Moriwaki, J.; Kimura, N.; Fujita, Y.; Fuji, S.; Hirano, T.; Koizumi, S.; Tsuge, T. The melanin biosynthesis genes of Alternaria alternata can restore pathogenicity of the melanin-deficient mutants of Magnaporthe grisea. Mol. Plant Microbe Interact. 1997, 10, 446-453. [CrossRef] [PubMed]

22. Veneault-Fourrey, C.; Barooah, M.; Egan, M.; Wakley, G.; Talbot, N.J. Autophagic fungal cell death is necessary for infection by the rice blast fungus. Science 2006, 312, 580-583. [CrossRef] [PubMed]

23. Talbot, N.J.; Ebbole, D.J.; Hamer, J.E. Identification and characterization of MPG1, a gene involved in pathogenicity from the rice blast fungus Magnaporthe grisea. Plant Cell 1993, 5, 1575-1590. [CrossRef] [PubMed]

24. Lorang, J.M.; Tuori, R.P.; Martinez, J.P.; Sawyer, T.L.; Redman, R.S.; Rollins, J.A.; Wolpert, T.J.; Johnson, K.B.; Rodriguez, R.J.; Dickman, M.B.; et al. Green fluorescent protein is lighting up fungal biology. Appl. Environ. Microbiol. 2001, 67, 1987-1994. [CrossRef] [PubMed]

25. Zhang, X.; Wang, L.; He, C.; Luo, H. An efficient transient mesophyll protoplast system for investigation of the innate immunity responses in the rubber tree (Hevea brasiliensis). Plant Cell Tiss. Org. 2016, 126, 281-290. [CrossRef]

(C) 2018 by the authors. Licensee MDPI, Basel, Switzerland. This article is an open access article distributed under the terms and conditions of the Creative Commons Attribution (CC BY) license (http:/ / creativecommons.org/licenses/by/4.0/). 